\title{
Fundus Autofluorescence after Half-Dose Photodynamic Therapy for Chronic Central Serous Chorioretinopathy
}

\author{
Kyoko Fujita $^{a}$ Yutaka Imamura $^{b}$ Kei Shinodac Soiti Matsumoto ${ }^{d}$ \\ Mitsuko Yuzawa ${ }^{\mathrm{e}}$
}

${ }^{a}$ Department of Ophthalmology, Aichi Medical University, Aichi, Japan; ${ }^{b}$ Department of Ophthalmology, Teikyo University School of Medicine, University Hospital Mizonokuchi, Kanagawa, Japan; ' Department of Ophthalmology, Saitama Medical University, Saitama, Japan; ${ }^{d}$ Department of Ophthalmology, Teikyo University School of Medicine,

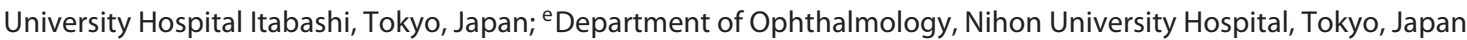

\section{Keywords}

Central serous chorioretinopathy · Fundus autofluorescence $\cdot$ Photodynamic therapy

\begin{abstract}
Introduction: To evaluate the fundus autofluorescence (FAF) images 1 year after half-dose photodynamic therapy (hdPDT) for chronic central serous chorioretinopathy (CSC). Methods: Forty-six eyes of 46 consecutive patients with chronic CSC underwent hdPDT. Short wavelength-elicited FAF images and enhanced depth imaging optical coherence tomographic (EDI-OCT) images were recorded before and at $1,3,6,9$, and 12 months after the hdPDT. The FAF images at 1 month were compared to those at 12 months after the hdPDT. Results: The serous retinal detachment (SRD) was resolved in all eyes. The best-corrected visual acuity (BCVA) improved significantly from $0.13 \pm 0.28$ logarithm of minimum angle of resolution (logMAR) units before to $0.01 \pm 0.17 \mathrm{log}$ MAR units at 12 months after the hdPDT ( $p=0.001$; paired $t$ test). The mean choroidal thickness decreased significantly from $365.4 \pm 103.0 \mu \mathrm{m}$ to $284.3 \pm 92.5 \mu \mathrm{m}$ at 12 months $(p<$ $0.001)$. Abnormal FAF images were present within the irradiated area in all the eyes before the hdPDT. In 5 of 46 eyes,
\end{abstract}

karger@karger.com www.karger.com/bmh

Karger $\stackrel{\text { ' }}{5}$

GOPEN ACCESS
(C) 2020 The Author(s)

Published by S. Karger AG, Basel

This is an Open Access article licensed under the Creative Commons Attribution-NonCommercial-4.0 International License (CC BY-NC) (http://www.karger.com/Services/OpenAccessLicense), applicable to the online version of the article only. Usage and distribution for commercial purposes requires written permission. identifiable changes of the FAF images were observed 12 months after hdPDT. None of the eyes had the confluent hypo-FAF type during the follow-up period. Univariate analyses showed that the choroidal thickness before hdPDT correlated significantly with hypo-FAF enlargement $(p=0.005)$. However, multivariate analyses showed that the association was not significant $(p=0.06)$. Conclusions: The progression of the RPE damages occurred in approximately one-tenth of the eyes that underwent hdPDT for chronic CSC. The longterm effect of progression of hypo-FAF on visual functions remains to be determined.

(C) 2020 The Author(s) Published by S. Karger AG, Basel

\section{Introduction}

Central serous chorioretinopathy (CSC) is characterized by leakages from the retinal pigment epithelium (RPE) and serous retinal detachment (SRD). In addition, the choroid is thickened as detected by enhanced-depth imaging optical coherence tomography (EDI-OCT) [1, 2]. Chronic CSC is often accompanied by degenerative changes of the RPE and atrophy of the sensory retina. 
Fundus autofluorescence (FAF) is a commonly used technique to detect the RPE changes which may be predictive of the changes in the visual functions [3].

We have recently reported the 1-year results of halfdose photodynamic therapy (hdPDT) for chronic CSC [4]. We reported that hdPDT was a safe and effective method to treat chronic CSC with a SRD. Most of our cases of chronic CSC had a resolution of the SRD and improvement of the best-corrected visual acuity (BCVA) [4].

We conducted our previous study to determine if hdPDT affected the RPE because RPE atrophy had been reported as a complication of PDT which can then lead to a reduction of vision after the treatment [5].

The purpose of this study was to evaluate the effect of hdPDT on the RPE. To accomplish this, we analyzed the changes in the FAF images of patients who had undergone hd-PDT.

\section{Materials and Methods}

The procedures used in this study were approved by the Institutional Review Board of the Nihon University Hospital (reference number: 151104), and they adhered to the tenets of the Declaration of Helsinki. A signed informed consent was obtained from all subjects for treatment and use of clinical data for research as a routine clinical procedure.

The diagnosis of chronic CSC was made by the presence of a SRD for at least 3 months, and the CSC was typically associated with atrophy and decompensation of the RPE as detected by fluorescein angiography (FA). The inclusion criteria were the presence of abnormally dilated choroidal vessels in the indocyanine green angiography (ICGA) images, and patients who were followed for at least 12 months. The exclusion criteria were patients who had other retinal diseases including age-related macular degeneration, polypoidal choroidal vasculopathy, pathologic myopia, and tilted disc syndrome. Patients who had undergone focal thermal laser photocoagulation or intravitreal injection of vascular endothelial growth factor (VEGF) inhibitors for the CSC within 3 months before PDT were excluded. Patients whose FAF images were not clear due to opacities of the ocular media were also excluded. All patients underwent a complete ophthalmologic examination including measurements of the BCVA, slit-lamp biomicroscopy, and dilated fundus examination. After pupillary dilation, the fundus of all subjects were photographed with a color fundus camera (TRC50IX; Topcon, Tokyo, Japan), and images of the retina were recorded by spectral-domain optical coherence tomography (SDOCT; HRA2, Heidelberg Engineering, Heidelberg, Germany). Shortwave length-elicited FAF images (SW-FAF; TRC-50IX; Topcon, Tokyo, Japan) were recorded before, 1, 3, 6, 9, and 12 months after the hdPDT. FA (TRC-50IX; Topcon, Tokyo, Japan) and ICGA (TRC-50IX; Topcon, Tokyo, Japan) were also performed before the hdPDT.

The subfoveal choroidal thickness was measured in the EDIOCT images by horizontal and vertical lines passing through the center of the fovea. The average of the horizontal and vertical subfoveal choroidal thickness was used in the statistical analyses.

The PDT was performed using half-dose verteporfin (Visudyne $^{\circledR}$; Novartis AG, Bulach, Switzerland). For this, $3 \mathrm{mg} / \mathrm{m}^{2}$ of verteporfin was infused over $10 \mathrm{~min}$, and the laser treatment was begun 15 min after beginning the infusion. The total light energy delivered to the area of hyperpermeability was $50 \mathrm{~J} / \mathrm{cm}^{2}$. The area of irradiation was set to overlap the hyperfluorescent area measured in the ICGA images recorded at the middle to late phase.

\section{FAF Image Analyses}

The hypo-FAF images were classified as either confluent or granular according to an earlier study analyzing FAF of the number of eyes with CSC [3]. Confluent hypo-FAF was defined as a round or ovoid area of absent-FAF larger than one-fourth of a disc diameter (DD) as described previously [3]. Granular hypo-FAF was determined to be present when there was a grainy or coarse area of decreased fluorescence compared with the normal surrounding areas [3]. The PDT spot was superimposed on the FAF images, and the whole area showing hypo-FAF was demarcated and the change in size was determined by visual inspection independently by 2 retina specialists (K.F. and Y.I.) in a masked fashion. Whenever there were discrepancies between the 2 retina specialists, a third specialist (K.S.) made the final decision. The change in size of the hypo-FAF area at 12 months after hdPDT was compared to that at 1 month after hdPDT. The changes in size were classified into 2 types: the diameter of the hypo-FAF area was enlarged by less than one-fourth of the $\mathrm{DD}$, and the diameter of the hypo-FAF area was enlarged by one-fourth of the DD or more.

The primary outcome measure was the presence of changes of the hypo-FAF images at 12 months after hdPDT relative to the FAF images at 1 month after hdPDT.

Because the preoperative SRD may mask the FAF findings at the RPE level, the FAF images at 1 month were used as the images at baseline.

\section{Statistical Analyses}

The decimal visual acuity was converted to the logarithm of minimum angle of resolution (logMAR) units for statistical analyses. The clinical factors between eyes with and without FAF changes at 12 months were analyzed with Fisher exact tests for non-numeric data and Student $t$ tests for numeric data. To explore factors associated with FAF change, Firth logistic regression was employed to analyze variables with $p<0.1$ in univariate analysis. Firth logistic regression was used to reduce biases of ordinal logistic regression in the analysis of rare events such as FAF change [6].

\section{Results}

Forty-six eyes of 46 consecutive patients with chronic CSC whose SRD was resolved 1 month after the hdPDT were studied. The mean $( \pm S D)$ age at the time of the hdPDT was $52.8 \pm 8.1$ years, with a range of $32-71$ years. There were 10 women $(21.7 \%)$ and 36 men (78.3\%). The average duration of the symptoms at the time of the hdPDT was $47.0 \pm 68.8$ months with a range 


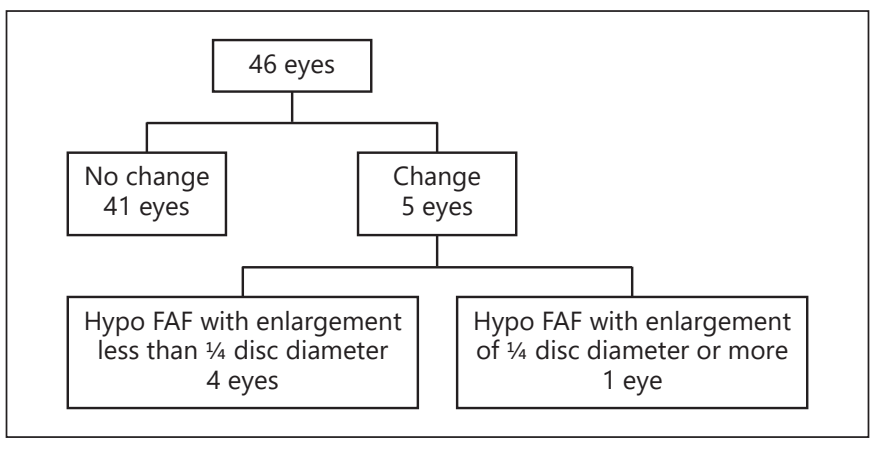

Fig. 1. Diagram showing the number of eyes with changes in the fundus autofluorescence within the irradiated area 12 months after half-dose photodynamic therapy.

of 6-360 months. One eye had undergone laser photocoagulation, 3 eyes had received anti-VEGF injections, and 4 eyes had received both laser photocoagulation and anti-VEGF injections before the hdPDT. Eight patients were using corticosteroids; 2 patients were using systemic corticosteroids, 1 patient was using inhalable corticosteroids, 3 patients were using skin cream, 1 patient was using nasal drops, and 1 patient had received a posterior subtenon triamcinolone acetonide injection at other hospitals. A history of steroid use was not available for 15 patients.

The ICGA images at the middle phase before the hdPDT showed that 37 eyes had intense hyperfluorescence and 9 eyes had intermediate hyperfluorescence. The mean spot diameter of the hdPDT was 5,589 $\pm 1,231 \mu \mathrm{m}$ with a range of 2,000-7,000 $\mu \mathrm{m}$. A SRD was present in the macula area in all eyes before the hdPDT, and all of the eyes had a resolution of the SRD after the hdPDT. None of the eyes had a recurrence of the SRD during the 12 months follow-up period.

Before the hdPDT, the mean BCVA was $0.13 \pm 0.28$ $\log$ MAR units. At 12 months, the mean BCVA improved significantly to $0.01 \pm 0.17 \log$ MAR units $(p=0.001)$. The mean choroidal thickness was $365.4 \pm 103.0 \mu \mathrm{m}$ before the hdPDT and it was significantly decreased to $284.3 \pm$ $92.5 \mu \mathrm{m}$ at 12 months after the hdPDT $(p=0.0001)$.

\section{FAF Outcomes}

All of the 46 eyes had abnormal FAF images within the irradiated areas before and 1 month after the hdPDT. At 12 months after the hdPDT, the FAF images were unchanged in 41 eyes, but 5 eyes showed enlargement of the hypo-FAF area compared to 1 month (baseline) (Fig. 1). There were no changes from normal to hyper- or hypo-

Fundus Autofluorescence after PDT for CSC
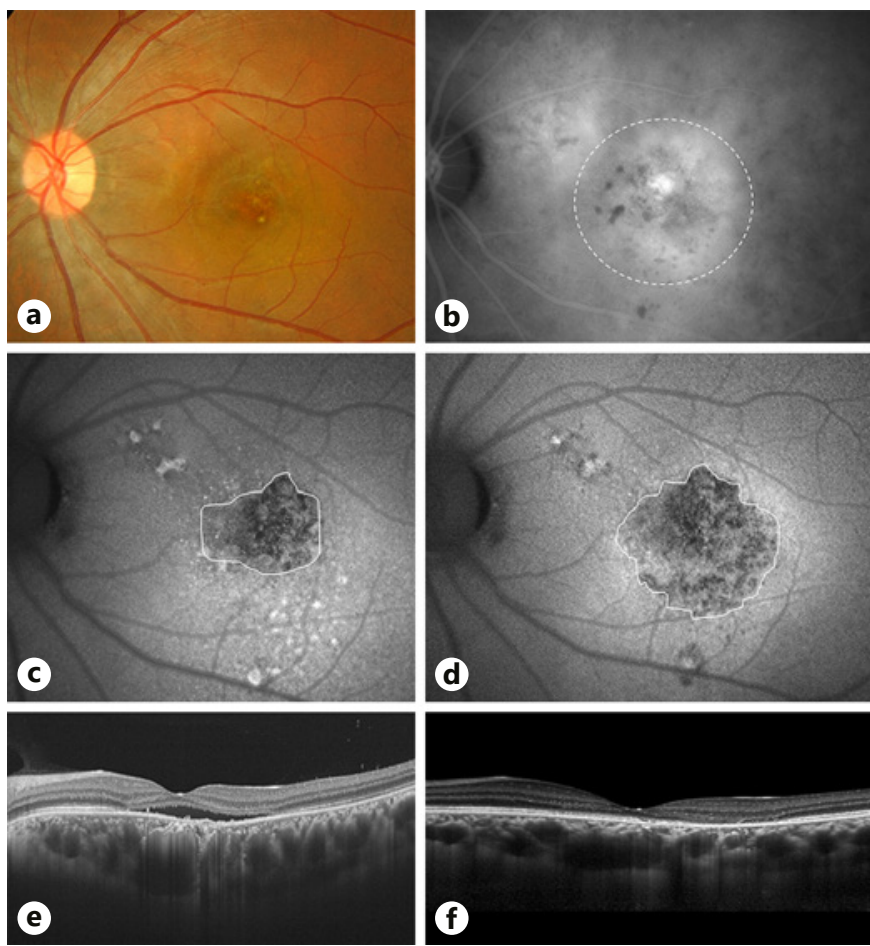

Fig. 2. Fundus photographs of a 32-year-old man with chronic central serous chorioretinopathy in the right eye. His best-corrected visual acuity was 0.52 logarithm of minimum angle of resolution units before the half-dose photodynamic therapy (hdPDT). a Color fundus photograph before hdPDT showing serous retinal detachment (SRD) at the macula. b Mid-phase indocyanine angiography before hdPDT showing choroidal vascular hyperpermeability. The white dotted circle indicates PDT spot. c One month after hdPDT, the fundus autofluorescence (FAF) shows granular hypo-FAF. The area surrounded by the white line indicates the hypo-FAF. $\mathbf{d}$ Twelve months after the hdPDT, granular hypo-FAF has enlarged by $1 / 4$ disc diameter or more. e Optical coherence tomography (OCT) image before hdPDT showing SRD involving the fovea. The subfoveal choroidal thickness was $614.5 \mu \mathrm{m}$. f OCT image at 12 months after hdPDT showing a normal foveal depression with resolution of SRD. The subfoveal choroidal thickness was $454.0 \mu \mathrm{m}$.

FAF. All eyes showing hypo-FAF at 1 and 12 months had granular type and none had confluent type.

Among the 5 eyes that had enlargement of the area of granular hypo-FAF at 12 months compared to 1 month after hdPDT, the size was enlarged by less than one-fourth of the DD in 4 eyes, and by one-fourth of the DD or more in 1 eye as shown in Figure 2.

The sex distribution, prior treatments for CSC, prior corticosteroid use, duration of the symptoms, PDT spot size, ICGA intensity, and BCVA did not differ significantly between the eyes with FAF changes and those with 
Table 1. Clinical findings with or without FAF changes

\begin{tabular}{|c|c|c|c|}
\hline & $\begin{array}{l}\text { No change in } \\
\text { FAF }(n=41)\end{array}$ & $\begin{array}{l}\text { Change in } \\
\text { FAF }(n=5)\end{array}$ & $p$ value \\
\hline Number of eyes & 41 & 5 & \\
\hline Age, years, mean $\pm \mathrm{SD}$ & $53.4 \pm 7.8$ & $48.4 \pm 9.1$ & $0.098^{\mathrm{a}}$ \\
\hline Male, $n(\%)$ & $31(75.6)$ & $5(100)$ & $0.27^{\mathrm{b}}$ \\
\hline History of treatment for CSC, $n$ & 7 & 1 & $0.63^{\mathrm{b}}$ \\
\hline History of corticosteroid use, $n$ & 7 & 1 & $0.45^{\mathrm{b}}$ \\
\hline Duration of symptoms, months, mean \pm SD & $39.1 \pm 50.1$ & $110.2 \pm 134.5$ & $0.175^{\mathrm{a}}$ \\
\hline PDT spot size, $\mu \mathrm{m}$, mean $\pm \mathrm{SD}$ & $5,517.0 \pm 1,240.6$ & $6,180.0 \pm 968.3$ & $0.133^{\mathrm{a}}$ \\
\hline ICGA intensity, intense: intermediate, $n$ & $33: 8$ & $4: 1$ & $0.68^{\mathrm{b}}$ \\
\hline Pre-PDT BCVA logMAR, mean \pm SD & $0.13 \pm 0.29$ & $0.12 \pm 0.20$ & $0.485^{\mathrm{a}}$ \\
\hline Pre-PDT choroidal thickness, $\mu \mathrm{m}$, mean \pm SD & $351.9 \pm 94.6$ & $476.1 \pm 101.9$ & $0.005^{\mathrm{a}}$ \\
\hline
\end{tabular}

Table 2. Multiple regression analysis of factors that correlate with fundus autofluorescence changes after half-dose photodynamic therapy

\begin{tabular}{llll}
\hline Variable & Odds ratio & $95 \%$ CI & $p$ value \\
\hline Age, years & 0.991 & $0.878-1.118$ & 0.881 \\
Choroidal thickness, $\mu \mathrm{m}$ & 1.011 & $0.999-1.023$ & 0.062 \\
\hline
\end{tabular}

CI, confidence interval.

no FAF changes. Univariate analyses showed that the preoperative choroidal thickness of the eyes with FAF changes was significantly thicker than those without (476.1 \pm $101.9 \mu \mathrm{m}$ vs. $351.9 \pm 94.6 \mu \mathrm{m} ; p=0.005$; Table 1$)$. However, the changes in choroidal thickness from baseline to 12 months after hdPDT was not significantly different between the eyes showing FAF changes and those without $(-0.24 \pm 0.12$ vs. $-0.22 \pm 0.12 ; p=0.690)$. At 12 months after hdPDT, the choroidal thickness of the eyes with FAF changes was significantly thicker than those without at 12 months $(356.3 \pm 87.7 \mu \mathrm{m}$ vs. $275.6 \pm 89.1 \mu \mathrm{m} ; p=0.033)$.

Multiple regression analysis showed that choroidal thickness before treatment did not correlate significantly with FAF change ( $p=0.06$; Table 2$)$.

\section{Discussion/Conclusion}

We compared the FAF findings at 1 month to those at 12 months after hdPDT in patients with chronic CSC. All of the 46 eyes had abnormal FAF before and at 1 month after the hdPDT. In 41 of the 46 eyes, the FAF findings at 12 months were the same as at 1 month, that is, no effect of the hdPDT. In 5 of 46 eyes, the FAF had a different appearance after the resolution of the SRD. It has been reported that RPE atrophy may develop after PDT in areas with previously normal RPE in the patients with chronic CSC [5].

FAF imaging is a method that allows a mapping of the lipofuscin deposits and their precursors in the retina and RPE. Lipofuscin accumulates in the RPE cells due to incomplete lysosomal degradation of photoreceptor outer segment disks [7]. Hyper-FAF is attributed to excessive accumulation of lipofuscin or its precursor above or within the RPE cells. Hypo-FAF mostly represents RPE atrophy. Histological examinations of human eyes have revealed dose-dependent damages of the choroidal vessels and the RPE after experimental PDT $[8,9]$.

In this study, 5 of 46 eyes showed hypo-FAF enlargement after hdPDT. One possible explanation for the enlargement is that although PDT reduces the choroidal thickness, the effect may not be sufficient in some cases, especially those with markedly thick choroid before PDT. In these cases, the choroid may remain thick and the pressure in the choriocapillaris may not be resolved. Pang et al. [10] suggested that a focal inward displacement of pachyvessels inflicts a pathogenic stimulus on the RPEBruch complex. Dansingani et al. [11] showed that dilated choroidal vessels existed at the level of choriocapillaris and loss of choriocapillaris occurred by the focal inward displacement of pachyvessels. Therefore, we supposed that a continuing compression of the RPE by a thick choroid may enhance the RPE damages. However, 
multivariate analyses showed that choroidal thickness before PDT did not correlate significantly with hypo-FAF enlargement $(p=0.06)$. Further studies with a larger number of cases are needed to confirm the results.

Another possible explanation for hypo-FAF enlargement was that the RPE damages were due to leakage of photosensitive molecules, for example, verteporfin from the choroidal vessels. Because the choroid is thicker in the area of choroidal vascular hyperpermeability on ICGA [12], the photochemical reactions may be stronger in eyes with a thickened choroid and subsequently RPE is damaged.

This study has several limitations. This was a retrospective study on a small number of patients. The evaluation of FAF was not quantitative. We are not aware of any report suggesting that RPE damages occurring after hdPDT have functional significance because the observations were limited to 1 year. However, we need to remember that visual disturbances can develop in eyes with CSC after hdPDT.

In conclusion, the RPE is affected in one-tenth of the eyes after undergoing hdPDT for chronic CSC. Although we did not have any complications associated with the RPE damages, the long-term significance of hdPDT-related RPE atrophy remains to be determined.

\section{Statement of Ethics}

The procedures used in this study were approved by the Institutional Review Board of the Nihon University Hospital (reference No. 151104), and they adhered to the tenets of the Declaration of Helsinki. A signed informed consent was obtained from all subjects before beginning the investigative procedures.

\section{Conflicts of Interest Statement}

The authors have no conflicts of interest to declare.

\section{Funding Sources}

No funding was received.

\section{Author Contributions}

K.F., Y.I., and K.S. clinically assessed, analyzed and interpreted the patient data regarding the ocular manifestations. K.F. and Y.I. drafted the article. K.S., S.M., and M.Y. provided critical revision and correction of the manuscript. All authors read and approved the final manuscript.

\section{References}

1 Gass JD. Pathogenesis of disciform detachment of the neuroepithelium. Am J Ophthalmol. 1967 Mar;63(3):1-139.

2 Imamura Y, Fujiwara T, Margolis R, Spaide RF. Enhanced depth imaging optical coherence tomography of the choroid in central serous chorioretinopathy. Retina. 2009 NovDec;29(10):1469-73.

3 Imamura Y, Fujiwara T, Spaide RF. Fundus autofluorescence and visual acuity in central serous chorioretinopathy. Ophthalmology. 2011 Apr;118(4):700-5.

4 Fujita K, Imamura Y, Shinoda K, Matsumoto CS, Mizutani Y, Hashizume K, et al. One-year outcomes with half-dose verteporfin photodynamic therapy for chronic central serous chorioretinopathy. Ophthalmology. 2015 Mar;122(3):555-61.
5 Cardillo Piccolino F, Eandi CM, Ventre L, Rigault de la Longrais RC, Grignolo FM. Photodynamic therapy for chronic central serous chorioretinopathy. Retina. 2003 Dec;23(6): 752-63.

6 Puhr R, Heinze G, Nold M, Lusa L, Geroldinger A. Firth's logistic regression with rare events: accurate effect estimates and predictions? Stat Med. 2017 Jun;36(14):2302-17.

7 Delori FC, Dorey CK, Staurenghi G, Arend O, Goger DG, Weiter JJ. In vivo fluorescence of the ocular fundus exhibits retinal pigment epithelium lipofuscin characteristics. Invest Ophthalmol Vis Sci. 1995 Mar;36(3):718-29.

8 Schlötzer-Schrehardt U, Viestenz A, Naumann GO, Laqua H, Michels S, Schmidt-Erfurth U. Dose-related structural effects of photodynamic therapy on choroidal and retinal structures of human eyes. Graefes Arch Clin Exp Ophthalmol. 2002 Sep;240(9):74857.
9 Schmidt-Erfurth U, Laqua H, SchlötzerSchrehard U, Viestenz A, Naumann GO. Histopathological changes following photodynamic therapy in human eyes. Arch Ophthalmol. 2002 Jun;120(6):835-44.

10 Pang CE, Freund KB. Pachychoroid neovasculopathy. Retina. 2015 Jan;35(1):1-9.

11 Dansingani KK, Balaratnasingam C, Naysan J, Freund KB. En face imaging of pachychoroid spectrum disorders with swept-source optical coherence tomography. Retina. 2016 Mar;36(3):499-516.

12 Maruko I, Iida T, Sugano Y, Ojima A, Sekiryu T. Subfoveal choroidal thickness in fellow eyes of patients with central serous chorioretinopathy. Retina. 2011 Sep;31(8):1603-8. 